MIT-CTP-3066

hep-ph/0101122

\title{
THEORETICAL OVERVIEW: MOTIVATIONS FOR LEPTON FLAVOR VIOLATION ${ }^{a}$
}

\author{
JONATHAN L. FENG ${ }^{b}$ \\ Center for Theoretical Physics, Massachusetts Institute of Technology \\ Cambridge, MA 02139 USA
}

\begin{abstract}
In the coming years, experiments underway will increase the sensitivity to charged lepton flavor violation by four orders of magnitude. These experiments will stringently probe weak scale physics. I review the status of global symmetries in the standard model and present several well-motivated models that predict observable lepton flavor violation. Finally, I describe what we might learn from future experimental results, whether positive or null.
\end{abstract}

\section{Introduction}

The diverse topics discussed at this conference, ranging from charged lepton flavor violation (LFV) to neutrino oscillations to proton decay, have been brought together by a common experimental dependence on high intensity muon and neutrino sources. From a theoretical perspective, however, these topics also have a unifying theme - they are all probes of global symmetries. The exploration of symmetries and symmetry breaking is, of course, central to particle physics. These experiments form a comprehensive program to probe the standard model's (broken) global symmetries, just as high energy colliders aim to study thoroughly the standard model's (broken) local symmetries.

Global symmetries are brittle. It is therefore somewhat surprising that global symmetry breaking is currently observed only in neutrino oscillations. In the near future, however, experiments will increase the sensitivity to LFV by four or more orders of magnitude. These experiments have the potential to uncover many exotic phenomena, which may exist at a variety of scales. 1 What is even more compelling, however, at least to me, is that these experiments will for the first time stringently probe the weak scale, where we have strong reasons to expect new physics to appear. There is thus the promise of a fascinating interplay between high precision LFV experiments and high energy experiments probing electroweak symmetry breaking. For this reason, results from upcoming LFV experiments, whether positive or null, will have interesting implications.

${ }^{a}$ Invited talk presented at New Initiatives in Lepton Flavor Violation and Neutrino Oscillations with Very Intense Muon and Neutrino Sources, 2-6 October 2000, University of Hawaii, USA.

${ }^{b}$ Electronic address: jlf@mit.edu 
I begin with a brief review of the standard model's global symmetries in Sec. 2. Section 3 contains a simple but telling model-independent analysis of the reach of present and future experiments. From this it will be clear that future LFV experiments will be strong probes of new physics at the weak scale. I will then focus on some broad possibilities related to the physics of electroweak symmetry breaking in Sec. 因, and consider the example of supersymmetry in Sec. 5. In Sec. 6, I conclude with several illustrations of what we might learn from future results.

\section{Global Symmetries of the Standard Model}

The fields of the standard model, their spins, and their quantum numbers under the three standard model gauge symmetries are:

$\begin{array}{ccccccc} & q & u & d & l & e & h \\ \mathrm{Spin} S & \frac{1}{2} & \frac{1}{2} & \frac{1}{2} & \frac{1}{2} & \frac{1}{2} & 0 \\ \mathrm{SU}(3) & 3 & 3 & 3 & 1 & 1 & 1 \\ \mathrm{SU}(2) & 2 & 1 & 1 & 2 & 1 & 2 \\ \mathrm{U}(1) & \frac{1}{6} & \frac{2}{3} & -\frac{1}{3} & -\frac{1}{2} & -1 & \frac{1}{2}\end{array}$

Renormalizability and gauge invariance restrict the interactions among these fields to the form

$$
\mathcal{L}_{\text {Yukawa }}=y_{\text {eij }} h \bar{l}_{i} e_{j}+y_{d i j} h \bar{q}_{i} d_{j}+y_{u i j} h^{c} \bar{q}_{i} u_{j},
$$

where $i$ and $j$ are generational indices. Through unitary rotations in generation space, we may set $y_{e i j}=\operatorname{diag}\left(y_{e}, y_{\mu}, y_{\tau}\right)$. The Yukawa couplings, then, conserve baryon number $B$, total lepton number $L$, and the lepton flavor numbers $L_{e}, L_{\mu}$, and $L_{\tau}$. These are the global symmetries of the standard model.

Global symmetries are broken in many possible ways, however. Beginning with the most robust theoretically, even in the standard model itself, nonperturbative quantum effects 2 break most of the global symmetries, preserving only $B-L$. Gravitational interactions are also expected to break all global symmetries through, for example, processes involving black holes. Both of these effects are typically extremely suppressed, but demonstrate that global symmetries are by no means sacred. More experimentally relevant examples arise in attempts to extend the standard model. In grand unified theories, for example, additional gauge bosons lead to global symmetry violation: in $\mathrm{SU}(5)$ only $B-L$ is preserved, and in $\mathrm{SO}(10)$, even this is broken. Finally, as we will see below, attempts to explain electroweak symmetry breaking often predict 
some form of global symmetry violation. In many cases, these predictions are generically at levels far beyond current constraints. It is a triumph of the standard model, and a source of frustration for those attempting to extend it, that its 'accidental' global symmetries are so accurately obeyed in nature.

\section{Mass Scales and Experimental Probes}

What is the current experimental status of global symmetries? For a modelindependent answer, we turn to the formalism of effective field theory. If we expect the standard model to be valid up to some energy scale $M$, the effects of physics above this scale may be accounted for by shifts in the standard model couplings and a series of non-renormalizable terms suppressed by powers of $M$. 3 The terms of Eq. (2) are then to be viewed as merely the first in a series. At higher orders in $1 / M$, one finds non-renormalizable operators that violate the global symmetries:

$$
\begin{array}{ll}
B \text {-violating: } & \frac{q q q l}{M^{2}}, \ldots \\
L \text {-violating: } & \frac{h h l l}{M}, \ldots \\
L_{i} \text {-violating: } & \frac{h \bar{l} \sigma^{\alpha \beta} e F_{\alpha \beta}}{M^{2}}, \frac{\bar{l} l \bar{e} e}{M^{2}}, \ldots,
\end{array}
$$

where generational indices have been omitted. The experimental status of global symmetries may be roughly summarized by determining what scales $M$ are currently being probed.

The most stringent probe of baryon number violation is proton decay. Given the operator of Eq. (3), the proton lifetime is $\tau_{p} \propto M^{4}$. Current

bounds on the proton lifetime with respect to various decay modes are of order $\tau_{p} \gtrsim 10^{32}$ years and imply $M \gtrsim 10^{15} \mathrm{GeV}$.

Evidence for total lepton number violation is provided by non-vanishing neutrino masses. The operator of Eq. (44) yields a neutrino mass when electroweak symmetry is broken and the Higgs scalar obtains a vacuum expectation value. For $\langle h\rangle \sim m_{W}$ and $m_{\nu}^{2} \sim \Delta m_{\mathrm{atm}}^{2} \sim 10^{-3} \mathrm{eV}^{2}$, the scale of $L$ violation seen in neutrino oscillation experiments is $M \sim 10^{14} \mathrm{GeV}$.

Neutrino oscillations also provide evidence for LFV at high scales, but do not preclude much larger $L_{i}$-violating, but $L$-conserving, effects in the charged lepton sector. For charged LFV, there are a wide variety of probes 1 including $\mu \rightarrow e \gamma, \mu-e$ conversion, $\mu \rightarrow e e e, \tau \rightarrow \mu \gamma, Z \rightarrow \mu \tau$, $\mathbb{B} K \rightarrow \mu e$, $\mathrm{D} J / \Psi \rightarrow \tau \mu$, and others. All of these may be useful (see below). Here, let's concentrate on two of the most promising: $\mu \rightarrow e \gamma$, and $\mu-e$ conversion. To estimate the 
reach of current and future experiments, consider a refined version of the first operator of Eq. (5):

$$
\mathcal{L}_{12}=e \frac{g^{2}}{16 \pi^{2}} \frac{m_{\mu}}{M_{12}^{2}} \bar{\mu} \sigma^{\alpha \beta} \text { e } F_{\alpha \beta} .
$$

The additional factors reflect an expectation that the new physics enters at one-loop and is also suppressed by a chirality insertion proportional to $m_{\mu}$. With this parametrization, the rates for $\mu \rightarrow e \gamma$ and $\mu-e$ conversion are

$$
\begin{aligned}
\frac{B(\mu \rightarrow e \gamma)}{1.2 \times 10^{-11}} & =\left[\frac{20 \mathrm{TeV}}{M_{12}}\right]^{4} & & (\mathrm{MEGA}) \\
\frac{R(\mu N \rightarrow e N)}{6.1 \times 10^{-13}} & =\left[\frac{10 \mathrm{TeV}}{M_{12}}\right]^{4} & & (\text { SINDRUM }) \\
\frac{B(\mu \rightarrow e \gamma)}{1 \times 10^{-14}} & =\left[\frac{110 \mathrm{TeV}}{M_{12}}\right]^{4} & & (\mu e \gamma \text { at PSI }) \\
\frac{R(\mu N \rightarrow e N)}{5 \times 10^{-17}} & =\left[\frac{110 \mathrm{TeV}}{M_{12}}\right]^{4} & & (\mathrm{MECO}) \\
\frac{R(\mu N \rightarrow e N)}{1 \times 10^{-18}} & =\left[\frac{280 \mathrm{TeV}}{M_{12}}\right]^{4} & & \text { (PRISM) }
\end{aligned}
$$

In the first two lines the rates are normalized to the best current bounds from the MEGA and SINDRUM ${ }^{8}$ collaborations. The last three are normalized to the expected sensitivities of the $\mu^{+} \rightarrow e^{+} \gamma$ experiment at PSI 9 the MECO experiment at Brookhaven, 10 and the proposed KEK/JAERI joint project, PRISM 11 Here $R(\mu N \rightarrow e N) \equiv \Gamma(\mu N \rightarrow e N) / \Gamma(\mu N \rightarrow$ capture $)$, and I have assumed $R(\mu N \rightarrow e N) \approx B(\mu \rightarrow e \gamma) / 300$. The latter relation depends on the target nucleus $N 12$ but this approximation is adequate for our purposes. More importantly, I have assumed that the new physics enters solely through the operator of Eq. (6). The sensitivity of $\mu-e$ conversion relative to $\mu \rightarrow e \gamma$ may be very much greater in other cases.

From Eq. (7), two conclusions may be drawn. First, experiments underway will extend the sensitivity in mass scale probed by roughly one order of magnitude. This is remarkable, given that the rates scale as $1 / M^{4}$. Second, the near future sensitivity will be well above the weak scale. Many exotic particles may mediate LFV. These include extra families, $Z^{\prime}$ bosons, and extra scalars. All of these particles may exist at or above the weak scale, and so provide motivation for LFV experiments. 13

Particularly compelling, however, is that LFV experiments will soon stringently probe the weak scale. Current theories of electroweak symmetry break- 
ing, which are necessarily tied to the weak scale, are therefore of great relevance for LFV experiments. I will discuss some of these possibilities in the following section. Note that in this analysis, I have already included factors for loop and chirality suppression. In specific models these may be supplemented by additional suppressions from, for example, GIM-type cancellations or small intergenerational mixing angles. A more detailed analysis runs counter to the model-independent spirit of this section. In concrete examples, however, these factors typically serve only to reduce the $\mathcal{O}(100 \mathrm{TeV})$ reach to a more typical weak scale value of $\mathcal{O}(1 \mathrm{TeV})$, and observable LFV rates from weak scale physics are still predicted.

\section{$4 \quad$ LFV and Electroweak Symmetry Breaking}

The standard model and two possible frameworks for extending it nicely illustrate some of the possibilities for global symmetry violation:

$\begin{array}{cccc}\text { Example } & \text { Standard Model } & \text { Extra Dims } & \text { SUSY } \\ M_{\text {High }} & B, L, L_{i} & & B, L \\ M_{\text {Weak }} & & B, L, L_{i} & L_{i}\end{array}$

Here I have indicated the scales at which the various global symmetries are generically broken. $M_{\text {Weak }} \sim 100 \mathrm{GeV}$ is the scale of electroweak symmetry breaking. $M_{\mathrm{High}}$ represents some much higher scale, such as the scale of righthanded neutrinos $M_{N} \sim 10^{14} \mathrm{GeV}$, grand unification $M_{\mathrm{GUT}} \approx 2 \times 10^{16} \mathrm{GeV}$, or gravitational interactions $M_{\text {Planck }} \sim 10^{19} \mathrm{GeV}$.

As previously noted, in the standard model, even extended to include neutrino masses, all global symmetries are broken only at some high scale. In this case, LFV experiments are far from the sensitivity required, and the exploration of global symmetry violation will be confined to neutrino and proton decay experiments. Note that the observed neutrino mixings do induce charged LFV at the loop level. However, for $\Delta m_{\nu}^{2} \sim 10^{-3} \mathrm{eV}^{2}$, the induced LFV is $B\left(l \rightarrow l^{\prime} \gamma\right) \sim 10^{-48}$, far beyond reach.

There are, however, several reasons to expect the standard model to be incomplete. Prominent among these is the gauge hierarchy problem, but there are many others, such as the requirement of sufficient baryogenesis and the necessity of additional non-baryonic dark matter. In addition, if current indications for a Higgs boson with mass $m_{h} \approx 115 \mathrm{GeV}$ are valid, the standard model vacuum will be destabilized at energy scales $\sim 10^{6} \mathrm{GeV}$, indicating that some new physics must enter below this scale.14

Extra dimensions have been suggested as one way to address the gauge hierarchy problem.15 With extra dimensions, the gravitational force law is 
modified at distances small compared to the size of the extra dimensions. For particular choices of the number and size of the extra dimensions, the fundamental gravitational scale may be lowered to near the weak scale, translating the gauge hierarchy problem into a problem of hierarchies in length scales.

In such scenarios, one typically expects all global symmetries to be broken at $M_{\text {grav }} \sim M_{\text {Weak }}$, leading to, among other things, catastrophic proton decay. The challenge of finding an elegant and compelling solution to this difficulty, particularly one consistent with baryogenesis, is one of the important problems in this scenario.

It is possible, however, to assume that proton stability is achieved by some mechanism and consider the possibility of LFV at low scales. This approach has been taken by a number of authors 16 They find that in certain scenarios constraints from LFV are among the most stringent. Large rates at future experiments may therefore be expected in models with low scale gravity, especially if the gravity scale is not far above the weak scale.

In supersymmetry, a hierarchy between $B$ and $L$ violation on the one hand and $L_{i}$ flavor violation on the other arises as an immediate consequence of the basic motivations for supersymmetry. As is well-known, two of the most important phenomenological motivations for supersymmetry are the gauge hierarchy problem and the existence of dark matter. Supersymmetry stabilizes the gauge hierarchy by introducing at the weak scale a scalar superpartner $\tilde{f}$ for every standard model fermion $f$ (and also a fermionic superpartner for every standard model boson). These new states introduce many additional renormalizable and gauge-invariant interactions. In the notation of superfields, the terms analogous to the standard model's Yukawa terms of Eq. (2) are given by the superpotential

$$
\begin{aligned}
W= & y_{e} H_{d} L E+y_{d} H_{d} Q D+y_{u} H_{u} Q U \\
& +\lambda L L E+\lambda^{\prime} L Q D+\lambda^{\prime \prime} U D D .
\end{aligned}
$$

Here generational labels are suppressed, and each superfield contains both scalar and fermion fields: $F \supset(f, \tilde{f})$. Interactions are formed by choosing any combination of two fermions and one scalar from any term.

It is not difficult to see that these new interactions destroy the beautiful properties of the standard model with respect to global symmetries. In particular, the terms in the second line of Eq. (9) violate both $B$ and $L$. However, these terms also allow all superpartners to decay, destroying the possibility of supersymmetric dark matter. This important virtue may be preserved by requiring $R$-parity conservation, where $R \equiv(-1)^{B+L+2 S}$. This eliminates all interactions in the second line of Eq. (9), and so $B$ and $L$ are again broken only at a high scale. As we will see below, however, the remaining supersymmetric 
interactions still allow $L_{i}$-violating processes at the weak scale, leading to the hierarchy displayed in Eq. (8).

\section{LFV in Supersymmetry}

Because LFV experiments will probe weak scale effects, new physics that is intimately tied to the weak scale is of special importance. I now consider several examples in supersymmetry, which provides a concrete and quantitative framework for evaluating the prospects for LFV.

Supersymmetric theories are specified by the interactions of Eq. (9), and a number of supersymmetry breaking terms. Of the latter, the most important for our present purposes are slepton masses

$$
m^{2 L L} \tilde{l}_{i}^{*} \tilde{l}_{j}+m^{2 R R} \tilde{e}_{i}^{*} \tilde{e}_{j},
$$

where the $m^{2}$ are a priori arbitrary Hermitian matrices, and trilinear terms

$$
A_{e i j} h_{d} \tilde{l}_{i}^{*} \tilde{e}_{j}
$$

which, after electroweak symmetry breaking, couple left- and right-handed charged sleptons through the mass matrix $m^{2 L R}{ }_{i j} \equiv A_{e i j}\left\langle h_{d}\right\rangle$.

These terms lead to charged LFV. To see this, it is convenient to choose the basis in which the standard model leptons are rotated to diagonalize the lepton Yukawa coupling, and the sleptons are rotated to preserve flavor-diagonal gaugino couplings. No additional flavor rotations are then available to diagonalize the terms of Eqs. (10) and (11), and the off-diagonal elements in the slepton mass matrices mediate charged LFV. Define parameters $\delta_{i j}^{M N} \equiv m_{i j}^{2 M N} / \tilde{m}^{2}$, where $\tilde{m}^{2}$ is the representative slepton mass scale, $M, N=L, R$, and $i, j$ are generational indices. The branching ratios for $l \vec{l} l^{\prime} \gamma$, normalized to current bounds, 17 constrain these parameters through 18

$$
\begin{aligned}
& \frac{B(\mu \rightarrow e \gamma)}{1.2 \times 10^{-11}} \sim \max \left[\left(\frac{\delta_{12}^{L L, R R}}{2.0 \times 10^{-3}}\right)^{2},\left(\frac{\delta_{12}^{L R}}{6.9 \times 10^{-7}}\right)^{2}\right]\left[\frac{100 \mathrm{GeV}}{\tilde{m}}\right]^{4} \\
& \frac{B(\tau \rightarrow e \gamma)}{2.7 \times 10^{-6}} \sim \max \left[\left(\frac{\delta_{13}^{L L, R R}}{2.2}\right)^{2},\left(\frac{\delta_{13}^{L R}}{1.3 \times 10^{-2}}\right)^{2}\right]\left[\frac{100 \mathrm{GeV}}{\tilde{m}}\right]^{4} \\
& \frac{B(\tau \rightarrow \mu \gamma)}{1.1 \times 10^{-6}} \sim \max \left[\left(\frac{\delta_{23}^{L L, R R}}{1.4}\right)^{2},\left(\frac{\delta_{23}^{L R}}{8.3 \times 10^{-3}}\right)^{2}\right]\left[\frac{100 \mathrm{GeV}}{\tilde{m}}\right]^{4} .
\end{aligned}
$$

$\left.\overline{{ }^{c} \text { More precisely, they constrain }\left(\delta_{i j}^{M N}\right.}\right)_{\mathrm{eff}} \sim \max \left[\delta_{i j}^{M N}, \delta_{i k}^{M P} \delta_{k j}^{P N}, \ldots,(i \leftrightarrow j)\right]$. 
Here I have assumed the lightest neutralino to be photino-like with mass $m_{\tilde{\gamma}}^{2} / \tilde{m}^{2}=0.3$; the bounds are fairly insensitive to this ratio.

The bounds from all three decays are non-trivial, but are especially stringent for transitions between the first and second generations. Clearly, arbitrary slepton mass matrices are forbidden. This is a statement of the (leptonic) supersymmetric flavor problem. For supersymmetry to be viable, some structure in the supersymmetry breaking masses must be present. I now turn to a variety of possibilities and analyze the LFV consequences of each.

\section{$5.1 S U(5)$}

The unification of gauge couplings is a strong motivation to consider supersymmetric grand unified theories. In the case of SU(5), the fields of each generation are contained in two multiplets, the $\mathbf{1 0}$ and $\overline{\mathbf{5}}$, while the up- and down-type Higgs doublets are contained in the $\mathbf{5}_{H}$ and $\overline{\mathbf{5}}_{H}$ multiplets, respectively. The Yukawa couplings are given by the superpotential

$$
W_{\mathrm{SU}(5)}=y_{u i j} \mathbf{1 0}_{i} \mathbf{1 0}_{j} \mathbf{5}_{H}+y_{d i j} \overline{\mathbf{5}}_{i} \mathbf{1 0}_{j} \overline{\mathbf{5}}_{H} .
$$

What is the form of the scalar masses? A simple and conservative assumption is that they are $m_{0}^{2} \widetilde{\mathbf{1 0}}_{i}^{*} \widetilde{\mathbf{1 0}}_{i}+m_{0}^{2} \widetilde{\overline{\mathbf{5}}}_{i}^{*} \widetilde{\overline{\mathbf{s}}}_{i}$, universal, and therefore flavorconserving, at $M_{\text {Planck. }}$. However, to determine the physical consequences, we must evolve these to low energy scales. In the renormalization group (RG) evolution above the GUT scale, grand unified interactions will generate offdiagonal slepton masses. 19 This is easily seen by noting that $y_{d}$ may be diagonalized with rotations on the $\mathbf{1 0}$ and $\overline{\mathbf{5}}$ fields, but there is then no remaining freedom to diagonalize $y_{u}$. RG evolution will then generate mixing of the $\mathbf{1 0}$ representations, and since $\tilde{e}_{R}, \tilde{\mu}_{R}, \tilde{\tau}_{R} \in \mathbf{1 0}$, non-vanishing $\delta_{i j}^{R R}$ are generated. Detailed calculations show that for some parameter choices, $\mu_{L}^{-} \rightarrow e_{R}^{-} \gamma$ is al ready near experimental limits, and well within reach of future experiments.20 Note that the left-handed sleptons are not significantly mixed, and so the branching ratio for $\mu_{R}^{-} \rightarrow e_{L}^{-} \gamma$ is negligible.

\subsection{Right-handed Neutrinos}

A similar analysis may be applied to supersymmetric theories with righthanded neutrinos $N$. The leptonic superpotential is

$$
W_{N}=y_{e i j} H_{d} L_{i} E_{j}+y_{\nu i j} H_{u} L_{i} N_{j}+m_{N i j} N_{i} N_{j} .
$$

Assume again, conservatively, that the slepton masses are universal at the Planck scale. Rotations of the $L, E$, and $N$ multiplets may diagonalize $y_{e}$ and 
$m_{N}$, but then off-diagonal entries in $y_{\nu}$ remain. These will generate mixing in the $L$ representations through $\mathrm{RG}$ effects above the right-handed neutrino mass scale, and since $\tilde{e}_{L}, \tilde{\mu}_{L}, \tilde{\tau}_{L} \in L$, non-vanishing parameters $\delta_{i j}^{L L}$ are generated, which mediate $\mu_{R}^{-} \rightarrow e_{L}^{-} \gamma$. These are again within reach of future experimental sensitivities.21 Note that, in contrast to the case of SU(5), here no $\delta_{i j}^{R R}$ mixing is generated, and so $\Gamma\left(\mu_{L}^{-} \rightarrow e_{R}^{-} \gamma\right) \approx 0$.

\subsection{Flavor Symmetries}

In the previous two examples, the Yukawa couplings were taken to have the hierarchical form required by experiment, and the scalar masses were assumed to have a simple universal form, presumably following from some unspecified supersymmetry breaking mechanism. An orthogonal approach is try to understand both Yukawa couplings and scalar mass textures in terms of a single flavor symmetry. In this approach, one must first find flavor symmetries that reproduce all of the known lepton masses and neutrino parameters. Then, since these symmetries determine also the superpartner mass matrices, unambiguous predictions for charged LFV follow.

The existing data may be summarized as follows: letting $\lambda \sim 0.2$ be the small flavor breaking parameter, the charged lepton masses satisfy

$$
m_{\tau} /\left\langle h_{d}\right\rangle \sim \lambda^{3}-1, \quad m_{\mu} / m_{\tau} \sim \lambda^{2}, \quad m_{e} / m_{\mu} \sim \lambda^{3},
$$

and the neutrino data imply

$$
\begin{aligned}
& \sin \theta_{23} \sim 1, \quad \sin \theta_{13}<\lambda, \quad \sin \theta_{12} \sim \begin{cases}1 & \mathrm{MSW}(\mathrm{LA}), \mathrm{VO} \\
\lambda^{2} & \mathrm{MSW}(\mathrm{SA})\end{cases} \\
& \frac{\Delta m_{\nu 12}^{2}}{\Delta m_{\nu 23}^{2}} \sim \begin{cases}\lambda^{2}-\lambda^{4} & \mathrm{MSW} \\
\lambda^{8}-\lambda^{12} & \mathrm{VO}\end{cases}
\end{aligned}
$$

where MSW(LA,SA) and VO denote the various solar neutrino solutions.

These data cannot be reproduced by the simplest possibility, a single U(1) symmetry. In this case, the fermion mass matrices are determined by charge assignments $Q$ as

$$
m_{e i j} \sim\left\langle h_{d}\right\rangle \lambda^{Q\left(\bar{l}_{i}\right)+Q\left(e_{j}\right)+Q\left(h_{d}\right)}, \quad m_{\nu i j} \sim \frac{\left\langle h_{u}\right\rangle^{2}}{M_{N}} \lambda^{Q\left(l_{i}\right)+Q\left(l_{j}\right)+2 Q\left(h_{u}\right)},
$$

with similar expressions for the scalar masses. It is not difficult to show that these textures predict that highly mixed neutrinos have similar masses, contradicting the most straightforward interpretation of Eq. (16). 
However, the observed data may be explained by, for example, $Z_{n} \times \mathrm{U}(1)$ symmetries, two breaking parameters with opposite charges, or holomorphic zeros.22 In these models, the above-mentioned difficulty is almost always circumvented as follows: the $m_{\nu}$ matrix produces hierarchical neutrino masses, and the $m_{e}$ matrix generates large neutrino mixing by requiring that the gauge and mass eigenstates of the $l$ representations are related by large rotations. However, because the lepton doublets contains charged leptons in addition to neutrinos, these rotation also generates large misalignments between the charged leptons and sleptons, producing large charged LFV rates.23

It is also possible for the $m_{\nu}$ matrix to have a very special form such that it singlehandedly generates both large mixings and large hierarchies in neutrino masses. Such examples have been constructed 23 Typically, however, the former possibility is realized. Large angle neutrino solutions then imply large LFV rates well within reach of future $\mu \rightarrow e \gamma$ and $\mu-e$ conversion experiments.23 Conversely, if no signal is seen in future experiments, many supersymmetric flavor models for neutrino masses will be excluded.

\section{What Will We Learn?}

\subsection{Precision $L F V$}

Given the dramatic improvements expected at future experiments, it is possible not only that LFV will be discovered, but also that it will be discovered with large statistics, allowing precision studies. Comparisons of the different rates of, for example, $\mu \rightarrow e \gamma, \mu-e$ conversion, and $\mu \rightarrow$ eee will allow us to disentangle which of the various operators in Eq. (5) are contributing. 4

The distributions within one mode may also be informative. With polarized muons, angular distributions in $\mu \rightarrow e \gamma$, and possibly also in $\mu-e$ conversion, may be used to disentangle the two chirality components. This will be a powerful way to distinguish between different models, 25 as illustrated by the examples in Secs. 5.1 and 5.2 .

\subsection{Interplay with Colliders}

If LFV is discovered in the near future, it is quite possible that the LFVinducing particles will be produced directly at high energies. LFV effects from real particle production may then also be seen at colliders. In the case of supersymmetry, sleptons produced in one flavor eigenstate may oscillate to another before decaying, leading to anomalously large rates for $e \mu+X$ production, for example. 2 In the case of two generation mixing, the cross section for such processes in the simplest cases is $\sigma(e \mu) \propto \sin ^{2} 2 \theta_{12}\left(\Delta m_{12}^{2}\right)^{2} /\left[\left(\Delta m_{12}^{2}\right)^{2}+4 \tilde{m}^{2} \Gamma^{2}\right]$, 
where $\theta_{12}$ and $\Delta m_{12}^{2}$ are the mixing angle and mass splitting between the first two slepton generations, and $\tilde{m}$ and $\Gamma$ are the average slepton mass and decay width. The rate for low energy $\mu-e$ processes has a different dependence on mixing parameters, with $B(\mu \rightarrow e) \propto \sin ^{2} 2 \theta_{12}\left[\Delta m_{12}^{2} / \tilde{m}^{2}\right]^{2}$. Thus, the combination of high energy and high precision experiments may allow us to determine both mixing angles and mass splittings independently, which would be impossible with only one type of experiment.

\subsection{Null Results}

If no signal is seen, the implications depend on what is discovered at high energy colliders. If only a standard model-like Higgs boson is discovered there, the null LFV results will simply give powerful constraints on a variety of phenomena. Of course, in this case, large questions will remain concerning the physics of electroweak symmetry breaking.

More likely, some part of the physics of electroweak symmetry breaking will be uncovered at colliders. We have seen that, on very general grounds, theories of electroweak symmetry breaking often predict LFV effects within reach of the upcoming experiments. Null results from these experiments, then, will provide highly non-trivial guides to understanding these theories. In the case of supersymmetry, for example, null results may have far-reaching implications, excluding many currently attractive supergravity theories and favoring theories that generate extremely degenerate superpartners at a low scale.

\section{Acknowledgments}

I thank Y. Kuno and W. Molzon for the invitation to participate in this stimulating conference, and I am grateful to Y. Nir and Y. Shadmi for collaboration in the work described in Sec. 5.3. This work was supported in part by funds provided by the U. S. Department of Energy under cooperative research agreement DF-FC02-94ER40818.

\section{References}

1. For comprehensive reviews, see Y. Kuno and Y. Okada, hep-ph/9909265; and references therein.

2. G. 't Hooft, Phys. Rev. Lett. 37, 8 (1976); Phys. Rev. D 14, 3432 (1976).

3. T. Appelquist and J. Carazzone, Phys. Rev. D 11, 2856 (1975).

4. See, for example, J. I. Illana and T. Riemann, hep-ph/0010193; X. Bi, Y. Dai and X. Qi, hep-ph/0010270.

5. W. Molzon, these proceedings. 
6. See, for example, X. Zhang, hep-ph/0010105.

7. M. L. Brooks et al. [MEGA Collaboration], Phys. Rev. Lett. 83, 1521 (1999) [hep-ex/9905013.

8. P. Wintz, these proceedings.

9. J. Yashima, these proceedings.

10. J. Sculli, these proceedings; V. Tumakov, these proceedings.

11. M. Aoki, these proceedings; Y. Kuno, these proceedings.

12. See, for example, A. Czarnecki, W. J. Marciano and K. Melnikov, hepph/9801218; Y. G. Kim, P. Ko, J. S. Lee and K. Y. Lee, Phys. Rev. D 59, 055018 (1999) hep-ph/9811211; T. S. Kosmas, A. Faessler, F. Simkovic and J. D. Vergados, Phys. Rev. C 56, 526 (1997) nucl-th/9704021;

A. Czarnecki, these proceedings; T. S. Kosmas, these proceedings.

13. W. Marciano, these proceedings.

14. M. Sher, Phys. Rept. 179, 273 (1989).

15. I. Antoniadis, N. Arkani-Hamed, S. Dimopoulos and G. Dvali, Phys. Lett. B436, 257 (1998) hep-ph/9804398.

16. See, for example, A. E. Faraggi and M. Pospelov, Phys. Lett. B458, 237 (1999) hep-ph/9901299; Z. K. Silagadze, hep-ph/9907328; A. Ioannisian and A. Pilaftsis, Phys. Rev. D 62, 066001 (2000) [hep-ph/9907522]; R. Kitano, Phys. Lett. B481, 39 (2000) [hep-ph/0002279].

17. K. W. Edwards et al. [CLEO Collaboration], Phys. Rev. D 55, 3919 (1997); S. Ahmed et al. [CLEO Collaboration], Phys. Rev. D 61, 071101 (2000) [hep-ex/9910060].

18. F. Gabbiani, E. Gabrielli, A. Masiero and L. Silvestrini, Nucl. Phys. B477, 321 (1996) hep-ph/9604387.

19. R. Barbieri and L. J. Hall, Phys. Lett. B338, 212 (1994) hep$\mathrm{ph} / 9408406$.

20. J. Hisano, T. Moroi, K. Tobe and M. Yamaguchi, Phys. Lett. B391, 341 (1997) hep-ph/9605296; J. Hisano, D. Nomura, Y. Okada, Y. Shimizu and M. Tanaka, Phys. Rev. D 58, 116010 (1998) hep-ph/9805367; Y. Okada, these proceedings.

21. J. Hisano and D. Nomura, Phys. Rev. D 59, 116005 (1999) hep$\mathrm{ph} / 9810479$; D. Nomura, these proceedings.

22. See, for example, Y. Grossman, Y. Nir and Y. Shadmi, JHEP9810, 007 (1998) hep-ph/9808355; Y. Nir and Y. Shadmi, JHEP9905, 023 (1999) hep-ph/9902293.

23. J. L. Feng, Y. Nir and Y. Shadmi, Phys. Rev. D 61, 113005 (2000) hep-ph/9911370.

24. A. de Gouvea, S. Lola and K. Tobe, Phys. Rev. D 63, 035004 (2001) hepph/0008085; K. Tobe, hep-ph/0008075; K. Tobe, these proceedings. 
25. Y. Kuno and Y. Okada, Phys. Rev. Lett. 77, 434 (1996) hep$\mathrm{ph} / 9604296$.

26. N. Arkani-Hamed, H. Cheng, J. L. Feng and L. J. Hall, Phys. Rev. Lett. 77, 1937 (1996) hep-ph/9603431; ibid., Nucl. Phys. B505, 3 (1997) hep-ph/9704205; M. Tanaka, these proceedings. 